\title{
Introducing Lumis - A Novel EBSD Detector
}

\author{
Chris Stephens ${ }^{1}$ and Zoran Pesic ${ }^{1}$
}

${ }^{1}$ Thermo Fisher Scientific, Unit 24, Birches Industrial Estate, East Grinstead, UK

Lumis is a new EBSD detector from Thermo Scientific. It incorporates the latest CMOS sensor technology, advanced optics and uses new indexing algorithms for the analysis of electron backscatter patterns (EBSPs) at $10 \mathrm{pA}$ and below.

Recent developments in sensor technology have led to a generation of imaging devices with improved performance characteristics. The latest CMOS sensors now provide exceptional sensitivity even at high frame rates due to a high dynamic range, increased quantum efficiency and improved noise management. Novel readout approaches, e.g. multiple regions, also offer new image acquisition strategies. The gap between conventional and scientific CMOS has narrowed significantly, with the former offering lower costs and attractive form factors, suitable for many low light applications.

Thermo Fisher Scientific have developed a novel EBSD detector which incorporates a large format CMOS sensor and reverse zoom optics to complement and extend the in-built CMOS sensor binning. A high efficiency, octagonal scintillator converts the EBSP signal into light, which is focussed on to a usercontrolled region of the sensor by the high-quality reverse zoom optics. This allows a continuous choice of "binned" EBSP size and gives full flexibility over EBSP speed, quality and sensitivity.

With $>2$ million pixels, the detector is ideally suited to phase identification (figure 2) and HR-EBSD strain measurement applications [1] when run at low zoom \& binning. Driving the optics in the opposite direction and applying sensor binning allows high speed EBSD, at several thousand frames per second for reduced collection times and higher sample throughputs.

The advanced optics \& high sensor sensitivity also allow EBSPs to be acquired at ultra low probe currents, e.g. below 10pA. This is particularly important for beam sensitive / dose restricted materials, or where higher probe current may introduce contamination or drift. Figure 3 shows a background corrected Nickel EBSP acquired at $6 \mathrm{pA}$, which represents a significant improvement over existing EBSD detectors.

References:

[1] CrossCourt4 http://www.hrebsd.com/wp/crosscourt/ 


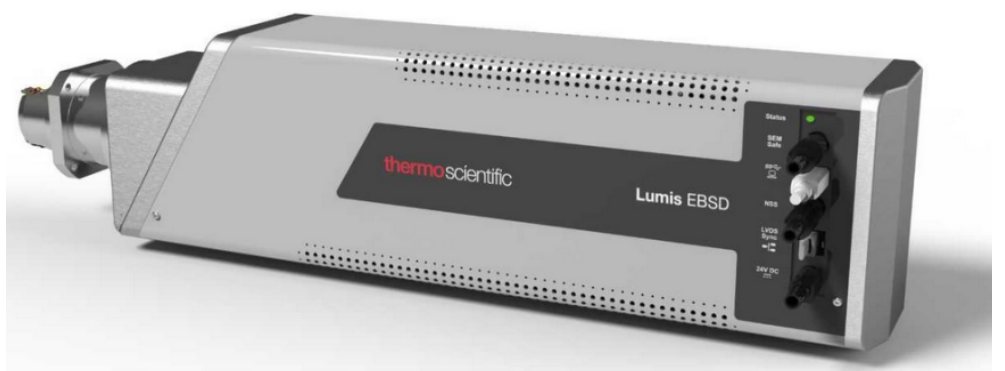

Figure 1. Image of the LUMIS EBSD detector.

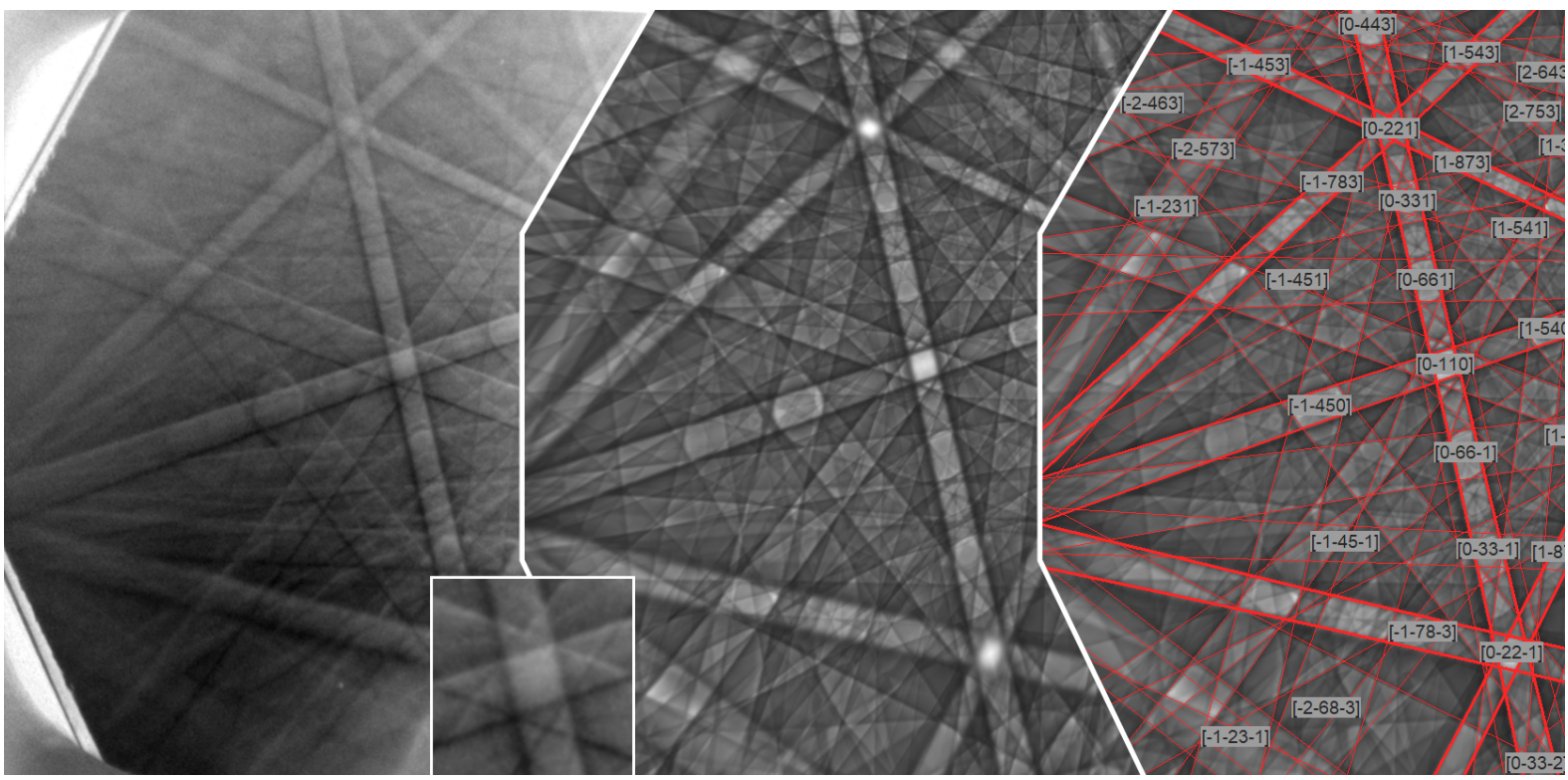

Figure 2. Hexagonal Iron (II) Sulphide, FeS, EBSP 30 kV; (lower left) detail of [0-110] zone; (middle) dynamical diffraction simulation, (right) indexed and labelled.

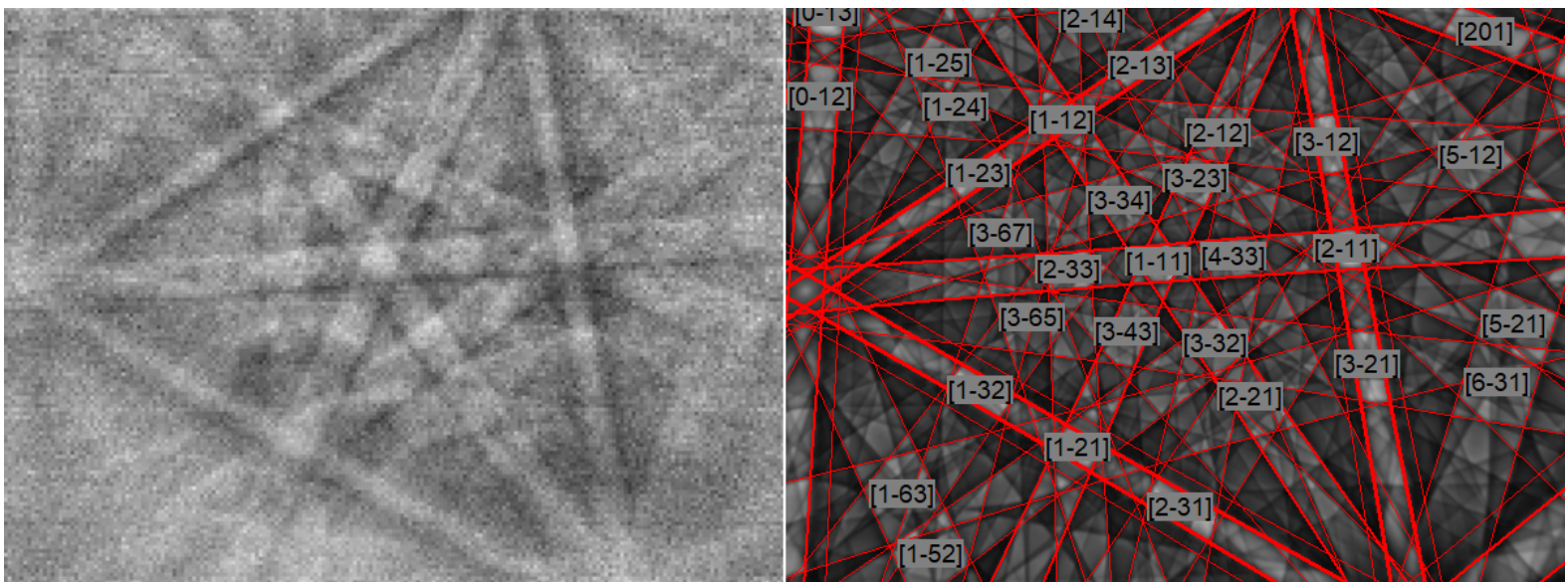

Figure 3. Experimental (left) and simulated (right) EBSPs of Ni with principle zone axes labelled. Acquired at $20 \mathrm{kV}$ and $0.006 \mathrm{nA}$. 